\title{
Magnetic and thermodynamic properties of $\mathrm{Nd}_{2} \mathrm{Ni}_{2} \mathrm{~Pb}$
}

\author{
V. Goruganti, Yang Li, Joseph H. Ross, Jr., and K. D. D. Rathnayaka \\ Department of Physics, Texas A\&M University, College Station, Texas 77843-4242 \\ Y. Öner \\ Department of Physics, Faculty of Sciences and Letters, Istanbul Technical University, \\ 34469 Maslak, Istanbul, Turkey
}

(Presented on 2 November 2005; published online 24 April 2006)

\begin{abstract}
We report magnetic and thermodynamic measurements for $\mathrm{Nd}_{2} \mathrm{Ni}_{2} \mathrm{~Pb}$, one of the recently discovered $R_{2} \mathrm{Ni}_{2} \mathrm{~Pb}$ family of layered intermetallics. A specific heat jump at $19 \mathrm{~K}$ corresponds to an antiferromagnetic transition involving crystal-field-split Nd levels. Magnetization measurements show this phase to have a canted structure, with a metamagnetic transition to an aligned phase occurring in $H=3 \mathrm{~T}$ at low temperatures. The single antiferromagnetic phase stands in contrast to the more complex magnetic structures and multiple transitions in the heavy-rare-earth members of this family. (C) 2006 American Institute of Physics. [DOI: 10.1063/1.2171958]
\end{abstract}

\section{INTRODUCTION}

Rare-earth intermetallic compounds have been the subject of many recent experimental investigations because of the variety of their magnetic properties. Recently, a series of ternary intermetallic compounds $R_{2} \mathrm{Ni}_{2} \mathrm{~Pb}$ was synthesized by Gulay et al. ${ }^{1}$ with $R=\mathrm{Y}, \mathrm{Sm}, \mathrm{Gd}, \mathrm{Tb}$, Dy, Ho, Er, Tm, and Lu. These plumbide 221 compounds crystallize in the $\mathrm{AlB}_{2} \mathrm{Mn}_{2}$-type structure, with the Cmmm space group (No. 65), and are interesting because of their unconventional magnetic ordering and multiple magnetic transitions. The crystal symmetry is highly anisotropic and possesses a layered structure. ${ }^{1}$ Giant magnetoresistance was observed in the Er, Ho, and Dy members of this series, ${ }^{2}$ and anisotropic magnetic properties in the $R=\mathrm{Gd}$ and Tb members. ${ }^{3} \mathrm{Nd}_{2} \mathrm{Ni}_{2} \mathrm{~Pb}$ was shown separately to form in the $R_{2} \mathrm{Ni}_{2} \mathrm{~Pb}$ structure. ${ }^{4} \mathrm{Fig}$ ure 1 shows the $\mathrm{Nd}_{2} \mathrm{Ni}_{2} \mathrm{~Pb}$ unit cell showing the naturally occurring multilayer with planes of $\mathrm{Nd}$ and $\mathrm{Pb}$ atoms and a wavy plane of $\mathrm{Ni}$ atoms. All $\mathrm{Nd}$ atoms in the unit cell are crystallographically equivalent. In this report we focus upon the low temperature magnetic properties of $\mathrm{Nd}_{2} \mathrm{Ni}_{2} \mathrm{~Pb}$ through magnetization and heat capacity measurements.

\section{EXPERIMENT}

Samples were prepared by arc melting, starting with the pure metals. The loss of $\mathrm{Pb}$ was accounted for by adjusting the starting ratio. Three samples, prepared both at Texas $A \& M$ and at Istanbul, showed nearly identical magnetic properties. One of these received a full $\mathrm{x}$-ray and electron microprobe analysis, and data for this sample are reported here. ${ }^{4}$ The sample was remelted several times to ensure homogeneity, then annealed at $873 \mathrm{~K}$ in an evacuated quartz tube for 30 days. X-ray diffraction was used to determine the crystal structure, with refinement performed using the General Structure Analysis System (GSAS) package. ${ }^{5}$ We used wavelength dispersive spectroscopy (WDS) scans to confirm the desired single phase with the 221 composition. Suscepti-

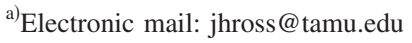

bility and magnetization measurements were done using a Quantum Design superconducting quantum interference device (SQUID) system. For these measurements the long axis of the sample was along the field direction, minimizing demagnetizing field effects. The temperature dependence of the heat capacity was measured by an adiabatic method in the temperature range from 2 to $300 \mathrm{~K}$, using a Quantum Design Physical Property Measurement System (PPMS) system.

\section{RESULTS AND DISCUSSION}

Powder x-ray analysis showed that the main phase is orthorhombic $\mathrm{Nd}_{2} \mathrm{Ni}_{2} \mathrm{~Pb}$ with $a=0.4138 \mathrm{~nm}, b=1.4292 \mathrm{~nm}$, and $c=0.3750 \mathrm{~nm}$, in agreement with established lattice parameters. ${ }^{4}$ Aside from the majority reflections, two additional peaks were seen; however, these were very weak and could not be indexed. There was no evidence for unreacted $\mathrm{Pb}$ or $\mathrm{NdNiPb}$ (111 phase) in the spectrum. WDS imaging

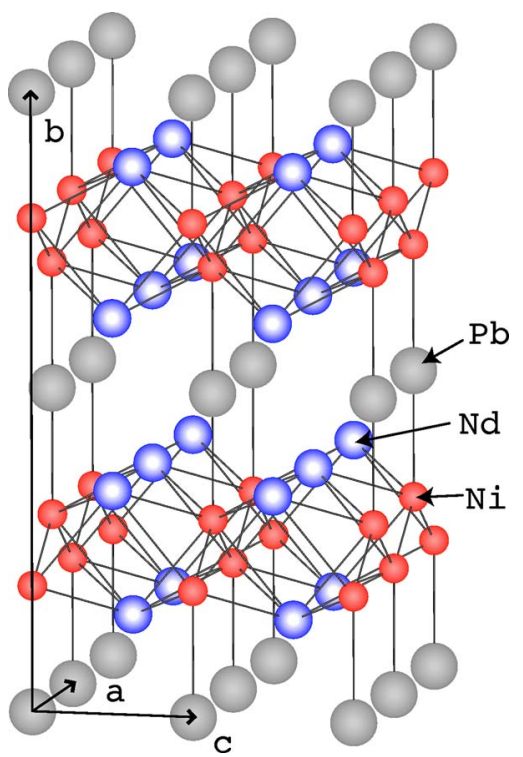

FIG. 1. (Color online) $\mathrm{Nd}_{2} \mathrm{Ni}_{2} \mathrm{~Pb}$ structure. Four cells are shown, viewed along the $a$ axis, showing the naturally occuring layered structure. 


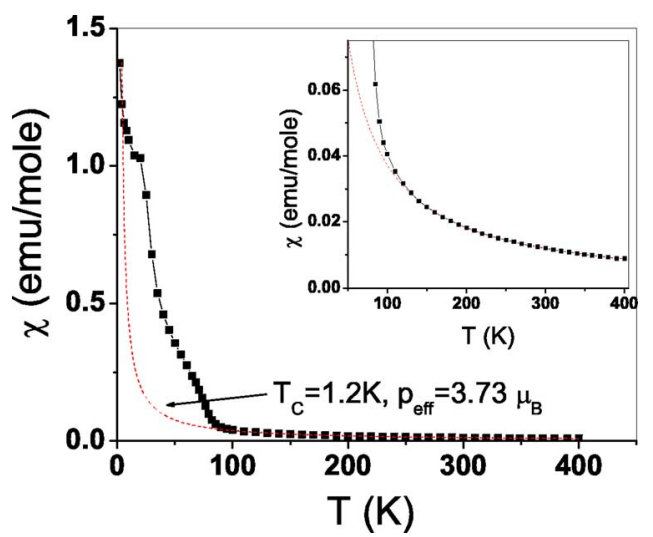

FIG. 2. (Color online) $\mathrm{Nd}_{2} \mathrm{Ni}_{2} \mathrm{~Pb}$ magnetic susceptibility measured in a field of $1 \mathrm{kOe}$, from 1.8 to $400 \mathrm{~K}$. Inset: High temperature data. Dashed line in both plots is a fit to the Curie-Weiss law.

showed a very small amount of 111 phase, below the x-ray detectability limit, but otherwise these scans confirmed the sample to be nearly pure $\mathrm{Nd}_{2} \mathrm{Ni}_{2} \mathrm{~Pb}$.

Figure 2 shows the dc susceptibility measured in a field of 1000 Oe. Anomalies are observed near 80 and $20 \mathrm{~K}$. A Curie-Weiss fit to the high-temperature data gives the dashed curve in Fig. 2. This yields $p_{\text {eff }}=3.73 \mu_{B}$, and $T_{p}=1.2 \mathrm{~K}$, with an effective moment close to the free ion moment for $\mathrm{Nd}^{3+}\left(p_{\text {eff }}=3.62 \mu_{B}\right){ }^{6}$ This establishes that $\mathrm{Nd}$ is in the trivalent state, and $\mathrm{Ni}$ is not magnetic as also observed in other $R_{2} \mathrm{Ni}_{2} \mathrm{~Pb}$ intermetallics. Below $80 \mathrm{~K}$, the susceptibility deviates from the Curie-Weiss behavior, due largely to crystalfield splitting of the $J=9 / 2 \mathrm{Nd}$ levels. We have observed some variability near this temperature for different samples, indicating that there may also be a trace magnetic phase involved. However, the magnetization and specific heat measurements described below make it clear that $\mathrm{Nd}_{2} \mathrm{Ni}_{2} \mathrm{~Pb}$ itself exhibits only a single magnetic transition, corresponding to the $20 \mathrm{~K}$ anomaly in the susceptibility.

Figure 3 shows the zero-field-cooled and field-cooled magnetizations at low temperatures. The development of hysteresis below the $20 \mathrm{~K}$ anomaly indicates that this phase has a net moment. However, the associated moment is quite small, indicating that this is likely a canted phase. Because of the lack of symmetry around the bonds joining rare-earth atoms in this structure, the Dzyaloshinsky-Moriya mechanism may drive parasitic ferromagnetism in a nominally antiferromagnetic system, ${ }^{7}$ likely explaining this behavior.

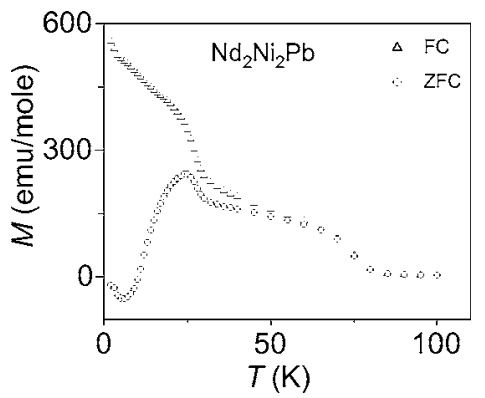

FIG. 3. Zero-field-cooled and field-cooled magnetizatations for $\mathrm{Nd}_{2} \mathrm{Ni}_{2} \mathrm{~Pb}$, showing irreversibility at low temperatures. Measuring and cooling fields of 50 Oe.

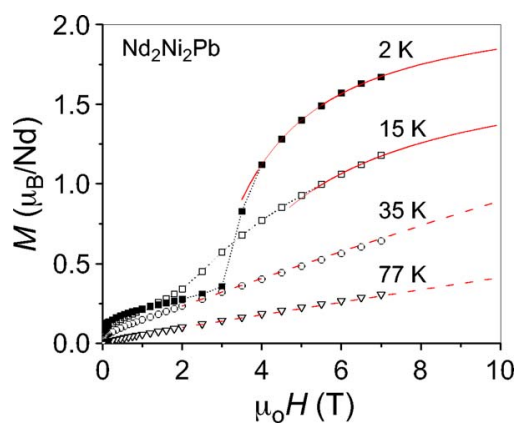

FIG. 4. (Color online) $M-H$ curve for $\mathrm{Nd}_{2} \mathrm{Ni}_{2} \mathrm{~Pb}$ measured in fields up to 7 $\mathrm{T}$, at the four temperatures illustrated. Solid curves: Saturation fit described in text. Dashed curves: Brillouin fits for 35 and $77 \mathrm{~K}$, showing paramagnetic behavior. Dotted curves for the 2 and $15 \mathrm{~K}$ data are guides to the eye.

In order to obtain more insight into the magnetic behavior, we carried out $M-H$ measurements. These are shown in Fig. 4. At $2 \mathrm{~K}$ a metamagnetic transition is seen at $3 \mathrm{~T}$ with large increase in magnetization. At $15 \mathrm{~K}$ the corresponding transition occurs at $1.5 \mathrm{~T}$. A fit to the $2 \mathrm{~K}$ saturation behavior, using a function of the form, ${ }^{7} \mathrm{M}=\mathrm{A}-\mathrm{B} / \mathrm{H}-\mathrm{C} / \mathrm{H}^{2}$ yielded a saturation magnetization corresponding to $2.1 \mu_{B}$ per $\mathrm{Nd}$. The fitted curve is shown in Fig. 4, along with a similar fitted curve for $15 \mathrm{~K}$. This saturation value is smaller than the free ion moment of $\mathrm{Nd}$; however, it is consistent with the range of values expected for a crystal-field-split ground-state doublet for orthorhombic $\mathrm{Nd}_{2} \mathrm{Ni}_{2} \mathrm{~Pb}$. Assuming this to be the case, we plotted Brillouin functions in Fig. 4 corresponding to one $2.1 \mu_{B}$ doublet per $\mathrm{Nd}$, for 35 and $77 \mathrm{~K}$. The result is in good agreement with the paramagnetic response at those temperatures. This is a strong indication that the observed metamagnetic transition corresponds to the full alignment of these crystal-field-split effective spins, rather than a transition to a new canted state. Thus, the magnetic behavior can be characterized by a single antiferromagnetic phase (with accompanying parasitic ferromagnetism as descibed above), having $T_{N}=19 \mathrm{~K}$, while the aligned phase is an extension of the paramagnetic phase. The single magnetic phase observed here stands in contrast to multiple magnetic phase transitions and more complicated spin structures, for the heavier-rareearth analogs of $\mathrm{Nd}_{2} \mathrm{Ni}_{2} \mathrm{~Pb}^{2,8}$

Figure 5 shows the temperature dependence of the spe-

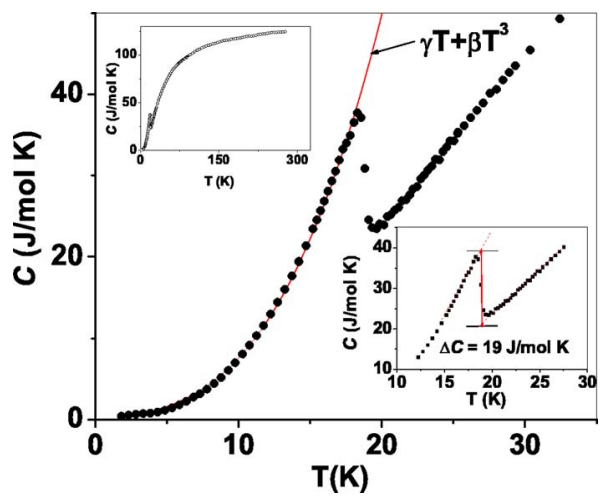

FIG. 5. (Color online) Low temperature heat capacity of $\mathrm{Nd}_{2} \mathrm{Ni}_{2} \mathrm{~Pb}$. Solid curve is the fit described in text. Upper inset: Heat capacity from 2 to $300 \mathrm{~K}$ shows classical saturated value of $125 \mathrm{~J} / \mathrm{mol} \mathrm{K}=3 R$ per atom. Lower inset: Specific heat jump at $19 \mathrm{~K}$ magnetic transition. 
cific heat. At $19.5 \mathrm{~K}$ there is a sharp $\lambda$-type singularity indicative of a cooperative phase transition. The specific heat jump at $T=T_{c}$ was found to be $C_{\exp }=19 \mathrm{~J} / \mathrm{mol} \mathrm{K}$. The theoretical specific heat jump at the transition temperature can be calculated from simple molecular field theory ${ }^{9,10}$ to yield $\Delta C=25 \mathrm{~J} / \mathrm{K} / \mathrm{mol}$ f.u., assuming that each spin is constrained to a pair of ground-state levels corresponding to an effective spin of $1 / 2$. This is consistent with the observed value if a reduction due to critical fluctuations above $T_{c}$ is taken into account.

Below $T_{c}$, a fit of the form $\gamma T+\beta T^{3}$ provides very good agreement, as shown in Fig. 5. The $T^{3}$ term is a characteristic signature of an antiferromagnetic phase $;{ }^{10}$ in this case $\beta$ contains both magnetic and phonon contributions, which are difficult to separate without further information on the magnitude of the phonon component. However, $\Delta S=\int C / T d T$, the total entropy change, can provide an upper bound on the magnetic contribution. The value thus obtained below $T_{c}$ is $\Delta S=16.5 \mathrm{~J} / \mathrm{mol} \mathrm{K}$, which can be compared to $\Delta S$ $=2 R \ln (2 J+1)=11.5 \mathrm{~J} / \mathrm{mol} \mathrm{K}$ for a magnetic disordering transition, for $2 \mathrm{Nd} /$ f.u., with the effective spin assumed to be $J=1 / 2$. A phonon contribution corresponding to the difference between these values is quite reasonable. On the other hand, ground-state multiplets corresponding to $J=1$ or greater give $\Delta S$ too large to be consistent with the observed entropy change. These results reinforce the conclusion obtained from magnetic measurements, indicating that $\mathrm{Nd}_{2} \mathrm{Ni}_{2} \mathrm{~Pb}$ exhibits a single magnetic transition having largely antiferromagnetic character, and show that the entire sample contributes to this transition.

\section{CONCLUSIONS}

We measured heat capacity, magnetization, and dc susceptibility for $\mathrm{Nd}_{2} \mathrm{Ni}_{2} \mathrm{~Pb}$. The heat capacity measurements indicate an antiferromagnetic phase transition at $19 \mathrm{~K}$ involving crystal-field-split levels. Magnetization measurements confirm this assignment and reveal a spin-alignment metamagnetic transition at $3 \mathrm{~T}$. The observation of a single antiferromagnetic phase differs from the observed behavior of the heavier-rare-earth $R_{2} \mathrm{Ni}_{2} \mathrm{~Pb}$ materials.

\section{ACKNOWLEDGMENTS}

This work was supported by the Robert A. Welch Foundation (Grant No. A-1526), the National Science Foundation (DMR-0103455 and DMR-0315476), and by the Texas A\&M University Telecommunications and Informatics Task Force.

${ }^{1}$ L. D. Gulay, Y. M. Kalychak, and M. Wolcyrz, J. Alloys Compd. 311, 228 (2000).

${ }^{2}$ A. D. Chinchure, E. Munoz-Sandoval, and J. A. Mydosh, Phys. Rev. B 66, 020409 (2002).

${ }^{3}$ E. Munoz-Sandoval, A. Diaz-Ortiz, A. D. Chinchure, and J. A. Mydosh, J. Alloys Compd. 369, 260 (2004).

${ }^{4}$ L. D. Gulay and M. Wolcyrz, Pol. J. Chem. 75, 1073 (2001).

${ }^{5}$ B. H. Toby, J. Appl. Crystallogr. 34, 210 (2001).

${ }^{6}$ C. Kittel, Introduction to Solid State Physics (Wiley, New York, 1986).

${ }^{7}$ S. Chikazumi, Physics of Ferromagnetism (Oxford University Press, New York, 1997).

${ }^{8}$ A. D. Chinchure, E. Munoz-Sandoval, and J. A. Mydosh, Phys. Rev. B 64, 020404 (2001).

${ }^{9}$ M. Endo, I. Umehara, Y. Adachi, Y. Isikawa, and K. Sato, Mater. Trans., JIM 41, 452 (2000).

${ }^{10}$ A. Tari, The Specific Heat of Matter at Low Temperatures (Imperial College Press, London, 2003). 